\title{
The Regional Difference of Relaxations Induced by Various Vasodilators in Isolated Dog Coronary and Mesenteric Arteries
}

\author{
Kunihisa MIWA* and Noboru TODA** \\ Department of Pharmacology. Shiga University of Medical Sciences. Seta. Ohtsu 520-21. Japan
}

Accepted April 8, 1985

\begin{abstract}
Relaxant responses to vasodilators, including nitroglycerin, sodium nitroprusside, prostaglandin $I_{2}$ sodium salt $\left(P \mathrm{Fl}_{2}\right)$, prostaglandin $E_{1}\left(P \mathrm{FE}_{1}\right)$. diltiazem hydrochloride and adenosine, were compared in helical strips of dog coronary arteries of different sizes and in coronary and mesenteric arterial strips. The relaxant responses to nitroglycerin, sodium nitroprusside, diltiazem and adenosine were significantly greater in coronary arteries than in mesenteric arteries, whereas the responses to $P \mathrm{PI}_{2}$ and $\mathrm{PGE}_{1}$ in these arteries did not significantly differ. In coronary arteries of different sizes, the relaxation induced by nitroglycerin was in the order of large > medium $>$ small-size, while in contrast, the relaxations by adenosine, $P G I_{2}$ and $P G E_{1}$ were greatest in the small-size arteries and least in the large-size arteries. The relaxant responses to sodium nitroprusside and diltiazem did not differ in the coronary arteries of different sizes. Nitroglycerin, sodium nitroprusside and diltiazem appear to dilate coronary arteries more predominantly than mesenteric arteries. The preferential dilator action of $\mathrm{PGI}_{2}$ and $P G E_{1}$ on distal coronary arteries, like that of adenosine, may lead more blood to re-distribute to the non-ischemic region of the heart in anginal patients.
\end{abstract}

Vasodilators have recently been used in patients with congestive heart failure to reduce systemic vascular resistance or left ventricular afterload with resultant improvement of left ventricular function (1, 2). Vasodilators have potential abilities to dilate not only the systemic vascular bed but also the coronary artery. It has been recognized that nitroglycerin acts mainly on the proximal, large coronary artery, whereas dipyridamole preferentially dilates the distal coronary artery, resulting in a decrease in total coronary vascular resistance $(3,4)$. However, there is little direct documentation concerning the regional difference in coronary artery responses to various vasodilator agents.

Thromboxane $A_{2} \quad\left(T X A_{2}\right)$ has been postulated to be one of vasoactive endogenous substances related to coronary

* Present Address: The 1st Department of Medicine. Fukui Medical School, 23-Shimoaizuki. Matsuoka, Yoshida-gun, Fukui 910-11. Japan

* To whom correspondence should be addressed. artery spasm (5). Carbocyclic TXA 2 (6), a stable analog of $T X A_{2}$, remarkably contracts isolated dog and monkey arteries (7). Accordingly, in isolated dog large, medium and small coronary arteries or in coronary and mesenteric arteries maximally contracted with carbocyclic $\mathrm{TXA}_{2}$, we compared the relaxant responses to nitroglycerin, sodium nitroprusside. prostaglandin $I_{2}$. prostaglandin $E_{1}$, diltiazem or adenosine.

\section{Materials and Methods}

Subjects: Mongrel dogs of either sex, weighing 6 to $21 \mathrm{~kg}$. were anesthetized with intravenous injections of pentobarbital in a dose of $30 \mathrm{mg} / \mathrm{kg}$ and were killed by bleeding from the common carotid arteries. The heart was rapidly removed, and left anterior descending and circumflex branches (epicardial location) of the left coronary artery of different sizes were isolated. Inside diameters were greater than $1.5 \mathrm{~mm}$ in large, proximal coronary arteries, 0.5 to $0.8 \mathrm{~mm}$ in the arteries of the medium-size arteries and 
less than $0.3 \mathrm{~mm}$ in small, dista! arteries. The mesenteric artery $(0.35$ to $0.5 \mathrm{~mm})$ was also resected. The arteries were cleaned and cut helically into strips, approximately $20 \mathrm{~mm}$ long. Endothelial cell functions were determined by testing a dose-dependent relaxation induced by acetylcholine (8). Each strip was fixed vertically between hooks in a muscle bath containing the nutrient solution which was aerated with a mixture of $95 \% \mathrm{O}_{2}$ and $5 \% \mathrm{CO}_{2}$ and maintained at $37 \pm 0.5^{\circ} \mathrm{C}$. The hook anchoring the upper end of the strips was connected to the lever of a forcedisplacement transducer (Nihon Kohden Kohgyo, Co., Tokyo, Japan). The maximum contraction was obtained at a passive tension of $1.5 \mathrm{~g}$ in strips of the medium-size coronary artery and the mesenteric artery (9) Cross-sectional areas of large. medium and small coronary arteries, calculated by the ratio of wet weight and length of strips, were $1.17 \pm 0.10 \mathrm{~mm}^{2} \quad(n=31), \quad 0.65 \pm 0.05 \mathrm{~mm}^{2}$ $(n=31)$ and $0.22 \pm 0.02 \mathrm{~mm}^{2} \quad(n=31)$. respectively (10). Therefore, the resting tension was adjusted to $3.0 \mathrm{~g}$ for large arteries, $1.5 \mathrm{~g}$ for medium-size arteries, $0.6 \mathrm{~g}$ for small arteries and $1.5 \mathrm{~g}$ for mesenteric arteries. Constitutions of the nutrient solution were as follows (mM): $\mathrm{Na}^{+}: 144.7, \mathrm{~K}^{\prime}: 5.4$, $\mathrm{Cl}^{-}:$131.5. $\mathrm{Ca}^{++}:$2.2. $\mathrm{Mg}^{++}:$1.0, $\mathrm{HCO}_{3}{ }^{-}$: 25.0, dextrose: 5.6. The $\mathrm{pH}$ of the solution was 7.25-7.35. Before the start of experiments, all preparations were allowed to equilibrate for $60-90 \mathrm{~min}$ in the control media, during which time the fluids were replaced every $10-15 \mathrm{~min}$.

Vasodilator dose-response curves: Isometric contractions and relaxations were displayed on an inkwriting oscillograph (Sanei Instrument Co., Tokyo). The contractile response to $\mathrm{KCl}(30 \mathrm{mM})$ was first obtained. the average values in large, medium and small coronary arteries being $2.07 \pm 0.25$ $(n=31), 2.93 \pm 0.31 \quad(n=31)$ and $2.46 \pm 0.38$ $(n=31) \mathrm{g} / \mathrm{mm}^{2}$ cross sectional areas, respectively. The preparations were then washed repeatedly and equilibrated for $30-$ $40 \mathrm{~min}$. Before the dose-response curve for vasodilators was obtained, arterial strips had been contracted with carbocyclic $\mathrm{TXA}_{2}$ $\left(10^{-7} \mathrm{M}\right)$, a stable analog of $\mathrm{TXA}_{2}$, in large, medium and small coronary arteries, and the contractions averaged $3.00 \pm 0.41 \quad(n=17)$. $3.81 \pm 0.60 \quad(n=17)$ and $2.48 \pm 0.53 \quad(n=17)$ $\mathrm{g} / \mathrm{mm}^{2}$ cross sectional area, respectively. There was no significant difference in the contractions of large, medium and small coronary arteries induced by $\mathrm{KCl}(30 \mathrm{mM})$ or carbocyclic $\mathrm{TXA}_{2}\left(10^{-7} \mathrm{M}\right)$. The vasodilators were added directly to the bathing media in cumulative concentrations. At the end of each series of experiments, papaverine in a concentration of $10^{-4} \mathrm{M}$ was added to attain the maximum relaxation. Relaxations induced by vasodilators relative to papaverine-induced relaxations are presented. The contraction induced by $\mathrm{KCl}$ (30 $\mathrm{mM}$ ) was taken as a standard for the contractile response. Paired comparisons were made in the responses to vasodilators of large, medium and small coronary arteries isolated from the same dogs. Paired comparisons in the responses of coronary arteries of medium-size and mesenteric arteries were also made.

Statistical analysis: All data are expressed as mean values \pm standard errors of the means. Statistical comparisons were performed by one-way analysis of variance with repeated measures and the Newman-Keuls test for multiple comparisons $(11,12)$ to evaluate possible differences between dose-response curves (Figs. 1, 2 and 5, and Table 1). Student's t-test was also used to determine the significance in maximum relaxations or median effective concentrations (ED50's).

Drugs: Drugs used were carbocyclic TXA (Ono Co., Osaka, Japan), nitroglycerin (Nihon Kayaku Co.. Tokyo, Japan), sodium nitroprusside (Nakarai Kagaku, Kyoto, Japan), $\mathrm{PGI}_{2}$ sodium salt (Ono Co.), $\mathrm{PGE}_{1}$ (Ono Co.), diltiazem hydrochloride (Tanabe Co., Osaka), adenosine (Kohjin Co., Tokyo) and papaverine hydrochloride.

\section{Results}

All six drugs including nitroglycerin, sodium nitroprusside, $\mathrm{PGI}_{2}, P G E_{1}$, diltiazem and adenosine relaxed dog coronary arteries of different sizes and mesenteric arteries in a dose-dependent manner, except for $P G E_{1}$ in low dose which contracted large coronary arteries. Nitroglycerin $\left(10^{-9}-10^{-5} \mathrm{M}\right)$, sodium nitroprusside $\left(10^{-9}-10^{-5} \mathrm{M}\right), \mathrm{PG} !_{2}\left(10^{-10}\right.$ 
$\left.10^{-6} \mathrm{M}\right) . \quad \mathrm{PGE}_{1}\left(10^{-7}-2 \times 10^{-6} \mathrm{M}\right)$ and adenosine $\left(10^{-8}-10^{-4} \mathrm{M}\right)$ rapidly relaxed the arteries: thus, a series for the doseresponse relationship was completed within 15 min. On the other hand, the diltiazem $\left(10^{-8}-10^{-4} \mathrm{M}\right)$-induced relaxation slowly developed; it took over $40 \mathrm{~min}$ to complete the dose-response relationship. This relaxation was sustained for over 1 hr. While relaxations induced by the other drugs were sustained only for a few minutes. Mean values of the apparent median effective concentration (ED50) of each drug in dog mesenteric arteries and coronary arteries of different sizes are summarized in Table 1.

Relaxant responses to vasodilators in coronary and mesenteric arteries: Doserelaxant response relationships of vasodilators in coronary and mesenteric arteries are shown in Figs. 1 and 2. Relaxant responses to nitroglycerin, sodium nitroprusside, diltiazem and adenosine were significantly larger in coronary arteries than in mesenteric arteries. Relaxant responses to $P G I_{2}$ and $P G E_{1}$ of these arteries did not significantly differ. Maximum relaxations induced by nitroglycerin, sodium nitroprusside and dittiazem were significantly larger $(P<0.01$ with nitroglycerin and sodium nitroprusside: $\mathrm{P}<0.02$ with diltiazem) in coronary arteries than in mesenteric arteries. ED50 values of nitroglycerin, sodium nitroprusside and adenosine were significantly less in coronary arteries than in mesenteric arteries (Table 1).
Relaxant responses to vasodilators in coronary arteries of different sizes: There were significant differences in the relaxant responses of large, medium and small coronary arteries to nitroglycerin, $\mathrm{PGI}, \mathrm{PGE}$ or adenosine. Typical recordings of relaxant responses to $\mathrm{PGl}_{2}$ are shown in Fig. 3 . Greater relaxations induced by $\mathrm{PGI}_{2}$ as well as adenosine are seen in the small-size arteries than in the large arteries. In contrast, nitroglycerin produced a greater relaxation in the large coronary artery, as shown in Fig. 4. Quantitative data on the relaxations of coronary arteries of different sizes are summarized in Figs. 5 and 6 . The ED 50 values of nitroglycerin were in the order of large $<$ medium<small coronary arteries, and the values of $\mathrm{PGI}_{2}$ and adenosine were in the order of large $>$ medium $>$ small coronary arteries (Tabie 1). PGE 1 in a low dose $\left(10^{-7}\right.$ M) contracted the large arteries and at the higher doses produced a relaxation. The administration of $\mathrm{PGE}_{1}$ elicited only relaxation in medium and small arteries. The maximum relaxation by $P G E_{1}$ was significantly larger $(P<0.05)$ in small coronary arteries than in large arteries. The ED50 value of $P G E_{1}$ was significantly larger $(P<0.05)$ in large coronary arteries than in small arteries (Table 1). There was no significant regional difference in the coronary artery relaxation induced by sodium nitroprusside and diltiazem.

Table 1. Mean values of apparent median effective concentration (ED50) of vasodilators

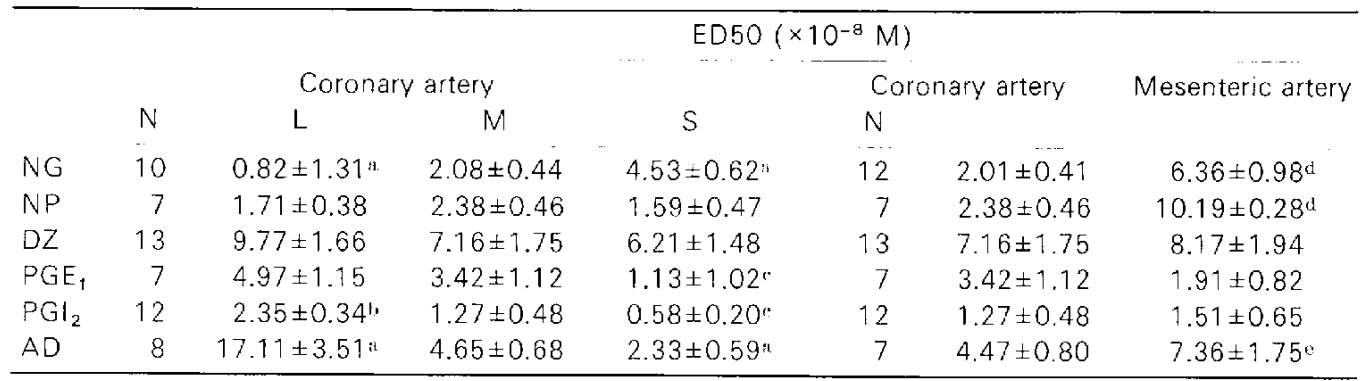

$N=$ number of preparations used, $L=$ large-size artery. $M=$ medium-size artery, $S=$ small-size artery. $N G=$ nigroglycerin. $N P=$ sodium nitroprusside, $D Z=$ diltiazem. $P G E_{1}=$ prostaglandin $E_{1}, \quad P G l_{2}=p_{0}-$ staglandin $i_{2}$. $A D=$ adenosine. Significantly different from values in medium-size artery: : $P<0.01$ : " $P<0.05$. Significantly different from values in large-size artery: e $P<0.05$. Significantly different from values in coronary artery: " $P<0.01$; $P<0.05$. Only the values significantly different by the Newman-Keuls test for multiple comparisons are included. 


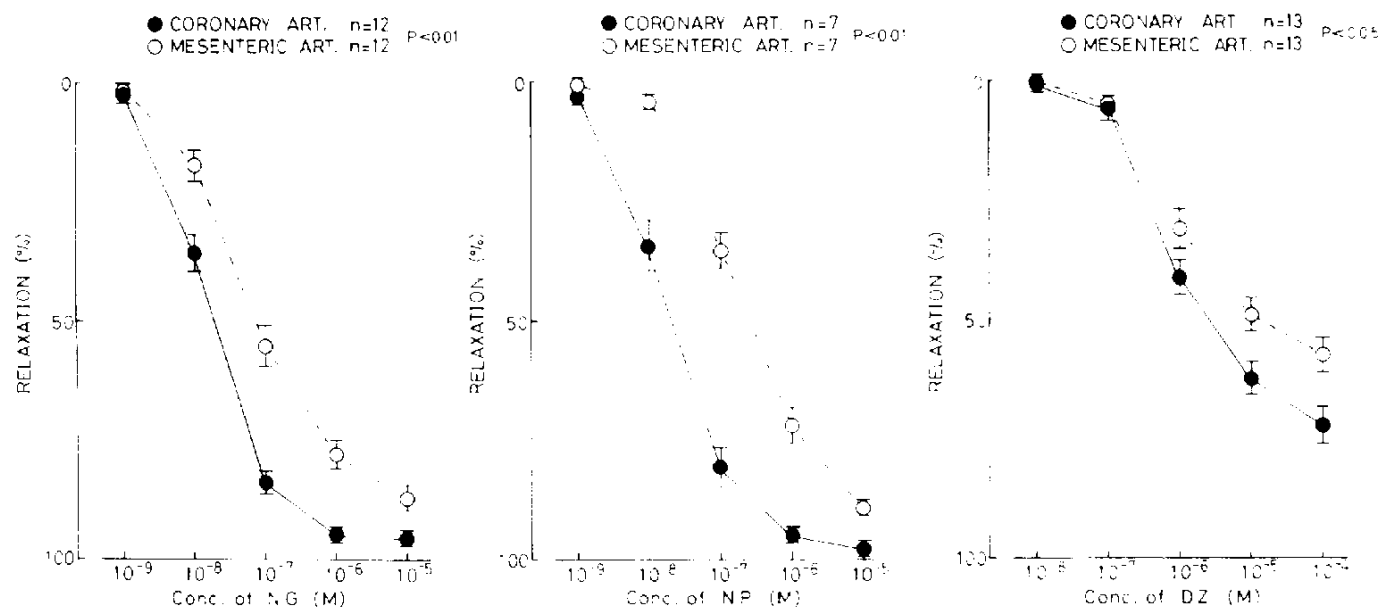

Fig. 1. Dose-response curves of nitroglycerin (NG), sodium nitroprusside (NP) and diltiazem (DZ) in isolated dog coronary and mesenteric arteries. Preparations were contracted with carbocyclic $\mathrm{TXA}_{2}\left(10^{-7}\right.$ M). Relaxation induced by $10^{-4} \mathrm{M}$ papaverine was taken as $100 \%$ : mean absolute values in coronary arteries were $1280 \pm 120 \mathrm{mg}(n=12), 1410=180 \mathrm{mg}(\mathrm{n}=7)$ and $1460 \pm 240 \mathrm{mg}(n=13)$. respectively, and those in mesenteric arteries were $1830 \pm 260 \mathrm{mg}(n=12), 1290 \pm 1100 \mathrm{mg}(n=7)$ and $1470 \pm 360 \mathrm{mg}$ $(n=13)$, respectively. Vertical bars represent the S.E.M. Relaxant responses induced by NG. NP and $D Z$ were significantly greater in coronary arteries ( $P<0.01$ with $N G$ and $N P, P<0.05$ with $D Z$ ) than in mesenteric arteries.
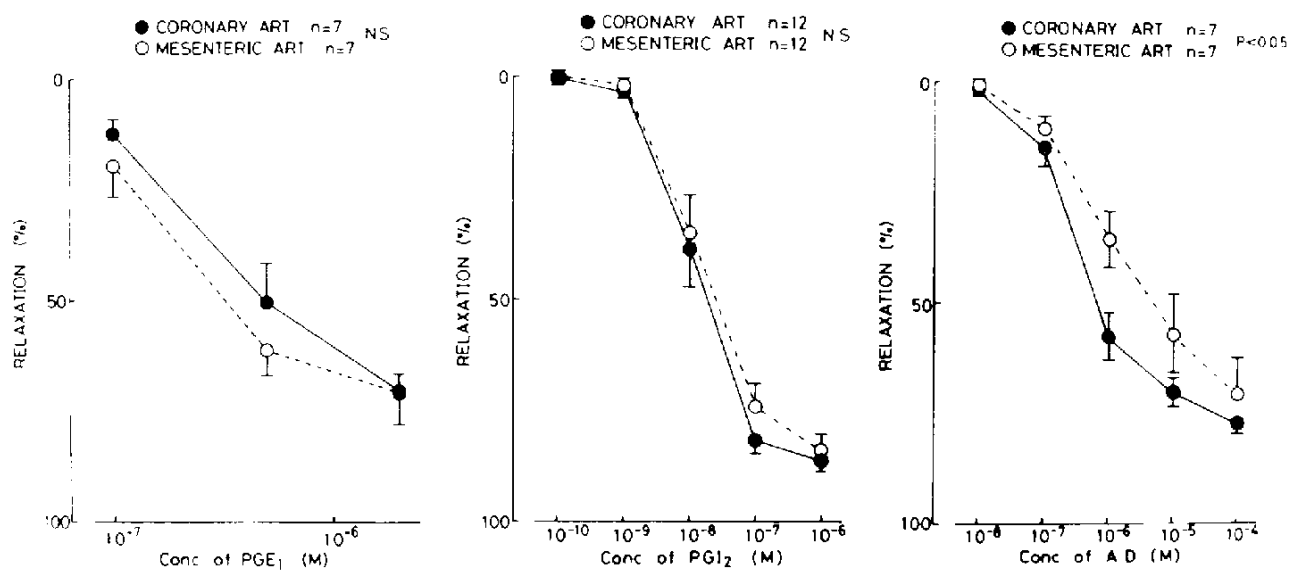

Fig. 2. Dose-response curves of $P G E_{1}, P G I_{2}$ and adenosine $(A D)$ in isolated dog coronary and mesenteric arteries. Preparations were contracted with carbocyclic $T X A_{2}\left(10^{-7} \mathrm{M}\right)$. Relaxation induced by $10^{-4} \mathrm{M}$ papaverine was taken as $100 \%$; mean absolute values in coronary arteries were $1330 \pm 160 \mathrm{mg}(n=7)$. $1620 \pm 230 \mathrm{mg}(n=12)$ and $1010 \pm 100 \mathrm{mg}(n=7)$, respectively, and those in mesenteric arteries were $1220 \pm 180 \mathrm{mg}(n=7), 1470 \pm 310 \mathrm{mg}(n=12)$ and $1520 \pm 200 \mathrm{mg}(n=7)$, respectively. Vertical bars represent the S.E.M. Relaxant responses induced by $A D$ were significantly greater in coronary arteries $(P<0.05)$ than in mesenteric arteries, but no significant differences were found in the relaxation induced by $P G E_{1}$ and $P G I_{2}$. 
RELAXATION BY PGI (C-TXA $_{2}$ CONTRACTED)

123

; 4

LARGE

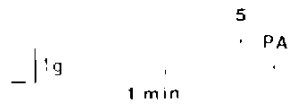

123

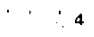

MEDIUM

$-19$

5 PA

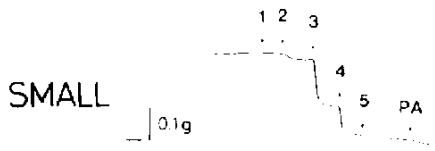

Fig. 3. Comparison of dose-related responses to $\mathrm{PGI}_{2}$ of dog coronary arterial strips of different sizes. Concentrations from 1 to $4: 10^{-9}, 10^{-8}, 10^{-7}$ and $10^{-6} \mathrm{M}$. respectively. Before the addition of $\mathrm{PGI}_{2}$, the strips were contracted with $10^{-7} \mathrm{M}$ carbocyclic $\mathrm{TXA}_{2}$. PA: $10^{-4} \mathrm{M}$ papaverine. The absolute values of the maximum relaxation in large, medium and smallsize arteries were 4630,2840 and $270 \mathrm{mg}$. respectively.
RELAXATION BY NG ( $C-T \times A_{2}$ CONTRACTED)

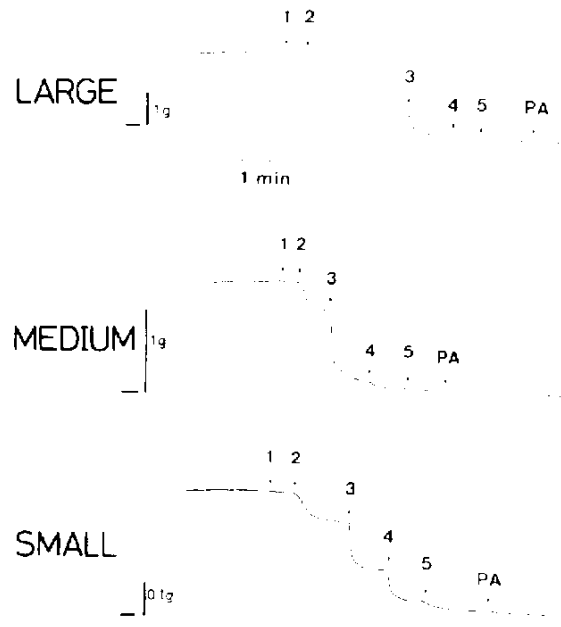

Fig. 4. Comparison of dose-related responses to nitroglycerin (NG) of dog coronary arterial strips of different sizes. Concentrations from 1 to 5 : $10^{-9}$. $10^{-8} \cdot 10^{-7} \cdot 10^{-6}$ and $10^{-5} \mathrm{M}$, respectively. Before the addition of nitroglycerin. the strips were contracted with $10^{-7} \mathrm{M}$ carbocyclic TXA 2 . PA: $10^{-4} \mathrm{M}$ papaverine. The absolute values of the maximum relaxation in large, medium and small-sizes arteries were 2090.1160 and $350 \mathrm{mg}$, respectively
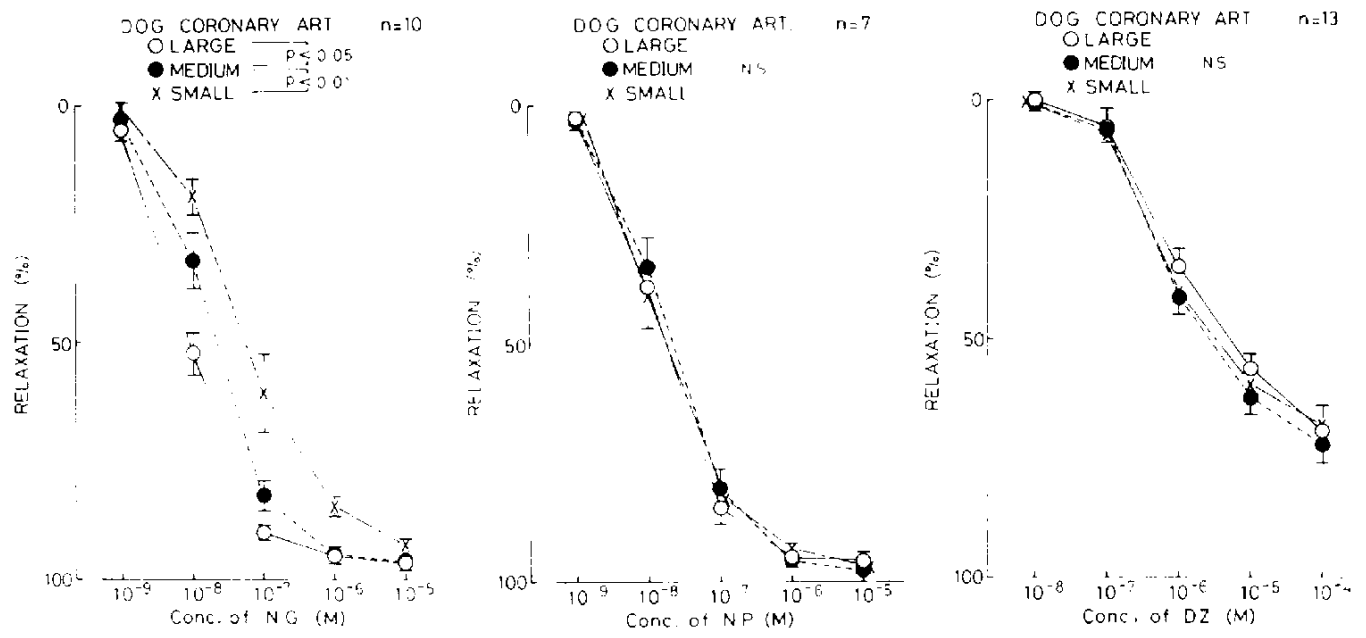

Fig. 5. Dose-response curves of nitroglycerin (NG), sodium nitroprusside (NP) and diltiazem (DZ) for isolated dog coronary arteries of different sizes. Preparations were contracted with carbocyclic $\mathrm{TXA}_{2}$ $\left(10^{-7} \mathrm{M}\right)$. Relaxation induced by $10^{-4} \mathrm{M}$ papaverine was taken as $100 \%$ : mean absolute values in large arteries were $3040 \pm 490 \mathrm{mg}(n=10), 3820 \pm 910 \mathrm{mg}(n=7)$ and $3210 \pm 430 \mathrm{mg}(n=13)$, respectively; those in medium-size arteries were $2020 \pm 430 \mathrm{mg}(n=10), 2440 \pm 330 \mathrm{mg}(n=7)$ and $2120 \pm 170 \mathrm{mg}$ $(n=13)$, respectively; and those in small-arteries were $560 \pm 110 \mathrm{mg}(n=10), 580 \pm 190 \mathrm{mg}(n=7)$ and $810 \pm 220 \mathrm{mg}(n=13)$, respectively. Vertical bars represent the S.E.M. Relaxant responses induced by NG were significantly greater in large arteries $(P<0.05)$ and less in small arteries $(P<0.01)$ than in medium arteries. There were no significant regional differences in the relaxations induced by NP and $D Z$. 

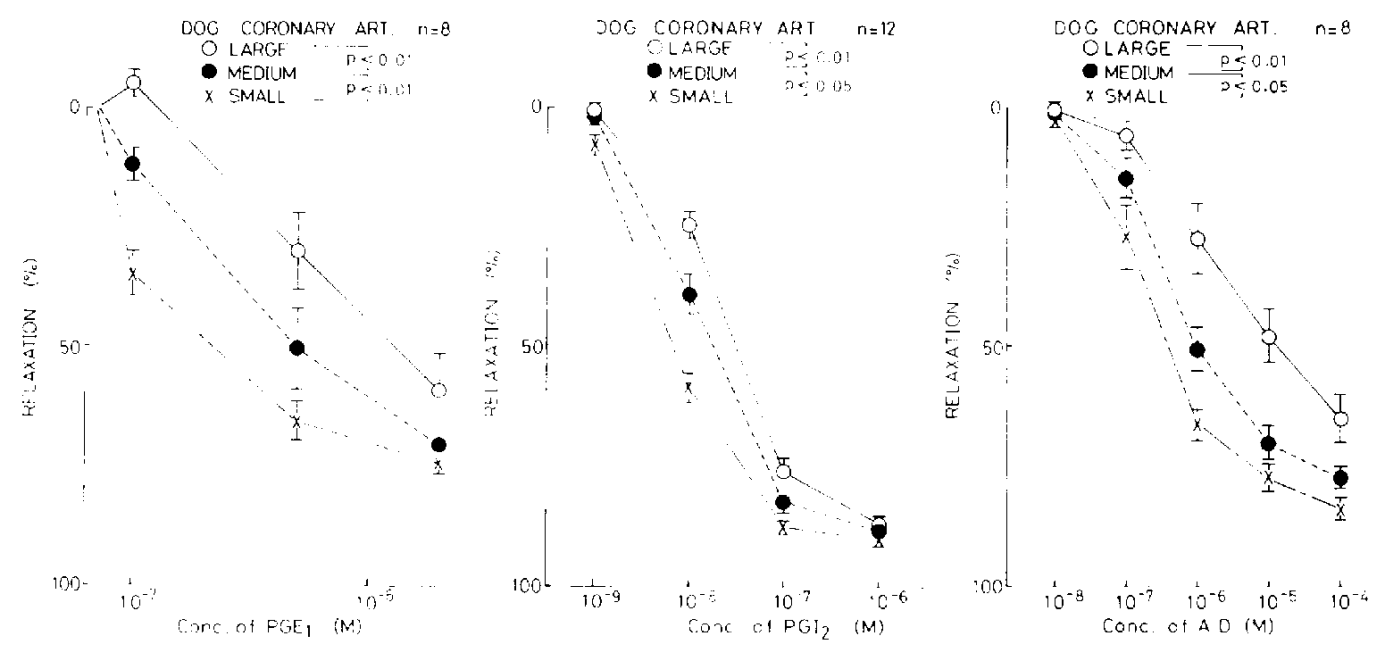

Fig. 6. Dose-response curves of $P G E_{1}, P G I_{2}$ and adenosine ( $A D$ ) in isolated dog coronary arteries of different sizes. Preparations were contracted with carbocyclic TXA $\left(10^{-7} \mathrm{M}\right)$. Relaxation induced by $10^{-4} \mathrm{M}$ papaverine was taken as $100 \%$ : mean absolute values in large arteries were $2430 \pm 610 \mathrm{mg}(\mathrm{n}=7)$, $3370 \pm 330 \mathrm{mg}(n=12)$ and $3460 \pm 650 \mathrm{mg}(n=8)$, respectively; those in medium-size arteries were 2020 $\pm 330 \mathrm{mg}(n=7), 2390 \pm 220 \mathrm{mg}(n=12)$ and $1870 \pm 170 \mathrm{mg}(n=8)$, respectively: and those in small arteries were $250 \pm 50 \mathrm{mg}(n=7), 790 \pm 190 \mathrm{mg}(n=12)$ and $280 \pm 40 \mathrm{mg}(n=8)$, respectively. Vertical bars represent S.E.M. Relaxant responses induced by $P G E_{1}, P G I_{2}$ and $A D$ were significantly greater in small arteries $\left(P<0.01\right.$ with $P G E_{1}, P<0.05$ with $P G I_{2}$ and $\left.A D\right)$ and less in large arteries $(P<0.01$ with $P G E_{1}, P G I_{2}$ and $\left.A D\right)$ than in medium-size arteries.

\section{Discussion}

The results of our experiments with isolated dog arteries revealed that some of the vasodilators produced a different magnitude of relaxations in the proximal, large coronary arteries and the distal, small arteries. Nitroglycerin dilated proximal coronary arteries to a greater extent than the distal arteries, whereas adenosine produced a greater relaxation in the distal arteries. These findings are consistent with those in the in vitro study of Schnaar and Sparks (13) using isolated dog coronary arteries of $2 \mathrm{~mm}$ o.d. as large arteries and arteries of $550 \mu$ o.d. as small ones. The inside diameters of small coronary arteries used in the present study are under 300 microns, being less than those used in the study of Schnaar and Sparks (13). Fam and McGregor (4) and Winbury et al. (3) aiso found similar results in cannulated large and small coronary arteries in in vivo dog experiments. Cohen and Kirk (14) using cannulated dog coronary vessels have shown that nitroglycerin preferentially reduces large coronary artery resistance under normal and ischemic conditions, and adenosine has a main action on resistive coronary vessels under normal perfusion, resulting in a reduction of the total coronary vascular resistance. Therefore, the isolated small coronary arteries that we used appear to share functional characteristics with resistant arterioles in vivo.

Drug-induced changes in large vessel tone would be important in patients with spasm of proximal coronary arteries. Coronary arterial spasm with or without fixed organic stenosis is considered to be closely related to the pathophysiology of not only variant angina but also of other ischemic heart disease including some of rest angina, effort angina and myocardial infarction (15-17). Mechanisms by which coronary arterial spasm is triggered are not fully known, but in recent days, great attention is paid to $\mathrm{TXA}_{2}$ derived from platelets in blood (5). Our results using coronary artery strips contracted with carbocyclic TXA 2 , a stable analog of $\mathrm{TXA}_{2}$ (6), indicate that nitro- 
glycerin may be most effective in remission of vasoconstriction in the large coronary arteries. On the contrary, exogenous $\mathrm{PGl}_{2}$ as well as $P G E_{1}$ do not appear to effectively inhibit coronary artery vasoconstriction. In fact, $\mathrm{PGI}_{2}$ given intravenously suppressed attacks evoked spontaneously or by ergonovine only in one out of 9 patients with variant angina (18)

Another clinical implication of the regional differences is related to the effects on the patients with ischemic heart disease who have significant organic stenosis in coronary arteries. Nitroglycerin is reported to dilate epicardial large coronary arteries and would contribute to the redistribution of the coronary blood flow (19-21). In contrast, adenosine. a potent metabolic coronary vasodilator. preferentially relaxed the distal portion (13. 22). The peripheral arteries which are expected to dilate maximally under the ischemic condition could not be dilated any more by vasodilators, while they dilate vessels in the healthy region, resulting in decrease of regional blood flow to ischemic areas (23, 24). Dipyridamole (25), an adenosine potentiator, is a drug potentially causing transient myocardial ischemia by such "coronary steal" due to its vasodilating action on coronary arterioles in patients with significant organic stenosis. Both $\mathrm{PGI}_{2}$ and $P G E_{1}$ may cause "coronary steal" because of their preferential actions on small arteries, like those of adenosine. Mehta et al. (26) reported that intravenous $P G l_{2}$ did not alter the regional blood flow across the fixed stenosis produced by an external occluder. but increased the flow in the non-ischemic area in dogs.

Both sodium nitroprusside and diltiazem did not show selective dilator actions on large and small coronary arteries. Diltiazem produced progressive and sustained relaxations, which were different from those induced by the other vasodilators used.

Our data also demonstrated that nitroglycerin, sodium nitroprusside, diltiazem and adenosine relaxed coronary arteries to a greater extent than mesenteric arteries when contracted with carbocyclic TXA 2 . Feldman et al. (27) reported that nitroglycerin in low doses dilated coronary arteries without influencing systemic blood pressure in man. Similar results with sodium nitroprusside were also obtained by Yeh et al. (28). Nakajima et al. (29) also demonstrated that in helical strips of rabbit coronary arteries, vasodilator action of diltiazem is more predominant than in the other arteries. The fact that the profound contraction induced by a high concentration of carbocyclic TXA was reversed by diltiazem predominantly in coronary arteries suggests that this $\mathrm{Ca}^{++}$ antagonist may be particularly effective in the treatment of coronary vasospasm. Both $\mathrm{PGI}_{2}$ and $P G E_{1}$ relaxed mesenteric arteries to a significantly greater extent than coronary arteries contracted with $\mathrm{PGF}_{2 \infty}(30)$. These data may indicate that $P \mathrm{PI}_{2}$ and $P G E_{1}$ contribute to a reduction of afterload.

We clearly demonstrated a regional difference in vasodilator actions in isolated dog arteries. Clinical evaluations are required to determine whether such a different action is true in patients with angina pectoris, acute heart failure and hypertension.

\section{References}

1 Cohn, J.N.: Vasodilator therapy for heart failure: The influence of impedence of left vetricular performance. Circulation 48, 5-8 (1973)

2 Massie, B.M. and Chatterjee, K.: Vasodilator therapy for acute and chronic heart failure. In Progress in Cardiology, Edited by Yu, P.N. and Goodwin. J.F.. Vol. 8, p. 197-234. Lea \& Febiger, Philadelphia (1979)

3 Winbury, M.M., Howe, B.B. and Hefner, M.A.: Effect of nitrates and other coronary dilators on large and small coronary vessels: A hypothesis for the mechanism of action of nitrates. J. Pharmacol. Exp. Ther. 168, 70-94 (1969)

4 Fam, W.M. and McGregor, M.: Effect of nitroglycerin and dipyridamole on regiona! coronary resistance. Circ. Res. 22, 649-659(1968)

5 Tada. M., Kuzuya, T., Inoue, M., Kodama, K., Mishima, M., Yamada, M., Inui, M. and Abe, H.: Elevation of thromboxane $B_{2}$ levels in patients with classical and variant angina pectoris. Circulation 64, 1107-1115 (1981)

6 Lefer, A.M., Smith, E.F., III. Araki, H., Smith, J.B., Aharony, D., Claremon, D.A., Magolda, R.L. and Nicolaou, K.C.: Dissociation of vasoconstrictor and platelet aggregatory activities of thromboxane by carbocyclic thromboxane $A_{2}$. a stable analog of thromboxane $A_{2}$. Proc. Natl. Acad. Sci. U.S.A. 77, 1706-1710 (1980) 
7 Toda, N.: Mechanism of action of carbocyclic thromboxane $A_{2}$ and its interaction with prostaglandin $\mathrm{I}_{2}$ and verapamil in isolated arteries. Circ. Res. 51, 675-682 (1982)

8 Furchgott, R.F. and Zawadzki, J.V.: The obligatory role of endothelial cells in the relaxation of arterial smooth muscle by acetylcholine. Nature 288, 373-376 (1980)

9 Toda, N., Hatano, Y. and Hayashi, S.: Modifications by stretches of the mechanical response of isolated cerebral and extracerebral arteries to vasoactive agents. Pfluegers Arch. 374, 73-77 (1978)

10 Miwa, K. and Toda, N.: Regional differences in the response to vasoconstrictor agents of dog and monkey isolated coronary arteries. Br. J. Pharmacol. 82, 295-301 (1984)

11 Winer, B.J.: Statistical Principles in Experimental Design. McGraw-Hill, New York (1971)

12 Zar, J.H.: Biostatistical Analysis. Prentice-Hali, Englewood Cliffs (1974)

13 Schnaar, R.L. and Sparks, H.V.: Response of large and small coronary arteries to nitroglycerin. $\mathrm{NaNO}_{2}$ and adenosine. Am. J. Physiol. 223. 223-228 (1972)

14 Cohen, M.V. and Kirk, E.S.: Differential response of large and small coronary arteries to nitroglycerin and angiotensin. Circ. Res. 33, 445-453 (1973)

15 Oliva, P.B., Potts, D.E. and Pluss, R.G.: Coronary arterial spasm in Prinzmetal's angina. Documentation by coronary arteriography. N. Engl. J. Med. 288, 745-751 (1973)

16 Oliva, P.B. and Breckinridge, F.C.: Arteriographic evidence of coronary arterial spasm in acute myocardial infarction. Circulation 56, 366374 (1977)

17 Yasue, H., Omote, S., Takizawa, A., Nagao, M., Miwa, $K$. and Tanaka, S.: Exertional angina pectoris caused by coronary arterial spasm: Effects of various drugs. Am. J. Cardiol. 43, 647-652 (1979)

18 Chierchia, S., Patrono, C., Crea, F., Ciabattoni, G., De Caterina, R., Cinotti, G.A., Distante, A. and Maseri, A.: Effects of intravenous prostacyclin in variant angina. Circulation 65, 470-477 (1982)

19 Vatner, S.F., Pagani, M., Manders, E.T. and Pasipoularides, A.D.: Alpha adrenergic vasoconstriction and nitroglycerin vasodilation of larger coronary arteries in the conscious dog. J. Clin. Invest. 65, 5-14 (1980)

20 Becker, L.C., Fortuin, N.J. and Pitt, B.: Effect of ischemia and antianginal drugs on the distribution of radioactive microspheres in the canine left ventricle. Circ. Res. 28, 263-269 (1971)

21 Mathes, P. and Rival, J.: Effect of nitroglycerin on total and regional coronary blood flow in the normal and ischaemic canine myocardium. Cardiovasc. Res. 5, 54-61 (1971)

22 Harder, D.R., Belardinelli, L., Sperelakis, N.. Rubio, R. and Berne, R.M.: Differential effects of adenosine and nitroglycerin on the action potentials of large and small coronary arteries. Circ. Res. 44, 176-182 (1979)

23 Cohen, M.V., Sonnenblick, E.H. and Kirk, E.S.: Coronary steal: Its role in detrimental effect of isoproterenol after acute coronary occlusion in dogs. Am. J. Cardiol. 38, 880-888 (1976)

24 Gallagher, K.P., Folts, J.D., Shebuski, R.J., Rankin, J.H.G. and Rowe, G.G.: Subepicardial vasodilator reserve in the presence of critical coronary stenosis in dogs. Am. J. Cardiol. 46, $67-73(1980)$

25 Becker, L.C.: Effect of nitroglycerin and dipyridamole on regional left ventricular blood flow during coronary artery occlusion. J. Clin. Invest. 58, 1287-1295 (1976)

26 Mehta, J., Nichols, W.W., Mehta, P., Pepine, C. and Conti, C.R.: Effects of prostacyclin on systemic and coronary hemodynamics in the dog. Am. Heart J. 102, 835-840 (1981)

27 Feldman, R.L., Pepine, C.J. and Conti, C.R.: Magnitude of dilatation of large and small coronary arteries of nitroglycerin. Circulation 64 , 324-333 (1981)

28 Yeh, B.K., Gosselin, A.J., Swaye, P.S., Larsen, P.B., Gentsch, T.O., Traad, E.A. and Faraldo, A.R.: Sodium nitroprusside as a coronary vasodilator in man. Am. Heart J. 93, 610-616 (1977)

29 Nakajima, H., Nosaka, K. and Hoshiyama, M.: Effects of diltiazem on the positive inotropic and vasoconstrictor responses to ouabain in vitro. Japan. J. Pharmaco!. 27, 910-914 (1977)

30 Toda, $\mathrm{N}$.: Responses to prostaglandin $\mathrm{H}_{2}$ and $\mathrm{I}_{2}$ of isolated dog cerebral and peripheral arteries. Am. J. Physiol. 238, H111-H117 (1980) 